\title{
BMJ Open Maternal multivitamin intake and orofacial clefts in offspring: Japan Environment and Children's Study (JECS) cohort study
}

\author{
Satomi Yoshida, ${ }^{1}$ Masato Takeuchi, ${ }^{1}$ Chihiro Kawakami, ${ }^{2}$ Koji Kawakami (D) , \\ Shuichi Ito, ${ }^{2}$ Japan Environment and Children's Study Group
}

To cite: Yoshida S, Takeuchi M, Kawakami C, et al. Maternal multivitamin intake and orofacial clefts in offspring: Japan Environment and Children's Study (JECS) cohort study. BMJ Open 2020;10:e035817. doi:10.1136/ bmjopen-2019-035817

- Prepublication history and additional material for this paper are available online. To view these files, please visit the journal online (http://dx.doi. org/10.1136/bmjopen-2019035817).

Received 18 November 2019 Revised 12 March 2020 Accepted 13 March 2020
Check for updates

(C) Author(s) (or their employer(s)) 2020. Re-use permitted under CC BY-NC. No commercial re-use. See rights and permissions. Published by BMJ.

\section{${ }^{1}$ Department of}

Pharmacoepidemiology, Graduate School of Medicine and Public Health, Kyoto University, Kyoto, Japan ${ }^{2}$ Graduate School of Medicine, Yokohama City University, Yokohama, Kanagawa, Japan

Correspondence to Dr Koji Kawakami; kawakami.koji.4e@kyoto-u. ac.jp

\section{ABSTRACT}

Objectives Orofacial clefts are common birth defects with a lack of strong evidence regarding their association with maternal nutrition. We aimed to determine whether a relationship exists between maternal nutrient or multivitamin intake and orofacial clefts.

Design This is a prospective, population-based nationwide cohort study.

Setting The study was conducted in 15 regional centres, consisting of local administrative units and study areas. Participants A total of 98787 eligible mother-child pairs of the Japan Environment and Children's Study were included.

Intervention Exposures were maternal nutrition and the use of supplemental multivitamins in mothers.

Primary and secondary outcome measures Outcomes were the occurrence of any orofacial cleft at birth. Multinomial logistic regression analyses were used to evaluate the association between maternal multivitamin intake and the incidence of orofacial clefts.

Results Of the 98787 children, $69(0.07 \%)$ were diagnosed with cleft lip alone, $113(0.11 \%)$ were diagnosed with cleft lip and palate, and $52(0.05 \%)$ were diagnosed with cleft palate within 1 month after birth. Regarding the total orofacial cleft outcome, statistically significant point estimates of relative risk ratios (RR) were determined for multivitamin intake before pregnancy ( $R R=1.71 ; 95 \% \mathrm{Cl} 1.06$ to 2.77$)$ and during the first trimester ( $\mathrm{RR}=2.00 ; 95 \% \mathrm{Cl} 1.18$ to 3.37), but the association was not significant for multivitamin intake after the first trimester ( $\mathrm{RR}=1.34 ; 95 \% \mathrm{Cl} 0.59$ to 3.01). Maternal micronutrient intake via food was not associated with the incidence of orofacial clefts in offspring.

Conclusions Intake of multivitamin supplements shortly before conception or during the first trimester of pregnancy was found to be associated with an increased incidence of orofacial clefts at birth. Pregnant women and those intending to become pregnant should be advised of the potential risks of multivitamin supplementation.

\section{INTRODUCTION}

Cleft lip (CL) and cleft palate (CP) are common birth defects, with a prevalence of approximately 1.7 per 1000 liveborn infants. ${ }^{1}$ In the USA, the estimated prevalence rates of
Strengths and limitations of this study

This study involved a dataset from a nationwide, birth cohort study.

- The data on maternal intake of multivitamin supplements and other drugs were based on interviews.

- We did not investigate the types of multivitamin supplements used, and therefore, the components and doses were unknown.

- The study outcome was the diagnosed occurrence of cleft lip (CL), cleft palate (CP) or CL with CP, which were evaluated within 1 month after birth by two paediatricians.

CL alone, CL with CP and CP alone were 3.1, 5.6 and 5.9 per 10000 live births, respectively. ${ }^{2}$ In contrast, the prevalence of CL with or without CP in Japan is 20.0 per 10000 births, which is approximately twice that reported in the USA, Canada and Australia. ${ }^{3}$ Furthermore, even greater geographical variations of 10-fold to 20-fold have been reported for the prevalence of CP at birth, ${ }^{1}$ although much of this variation is likely attributable to the difficulty in diagnosing some forms of CP in the immediate postnatal period.

Antiepileptic drugs, ${ }^{45}$ maternal smoking or secondhand smoke, ${ }^{6}$ and alcohol intake ${ }^{7}$ have been reported to increase the risk of orofacial clefts. Although folic acid is believed to have a preventive role against orofacial clefts, existing evidence remains generally inconsistent. ${ }^{89}$ A greater periconceptional maternal intake of nutrients predominantly from fruits and vegetables may help reduce the risk of orofacial clefts in the offspring, ${ }^{10}$ whereas a western dietary pattern may increase the risk of such defects. ${ }^{11}$ However, few epidemiological studies have demonstrated an association between maternal nutrition and orofacial clefts in human offspring; additional evidence from large cohort studies is needed. 
Our study aimed to examine the relationship between maternal nutrition and CL alone, CP alone and CL with $\mathrm{CP}$, with a focus on micronutrient and supplement intake in a Japanese, nationwide cohort.

\section{METHODOLOGY}

In January 2011, the Japanese Ministry of the Environment launched a large-scale epidemiological research project termed the Japan Environment and Children's Study (JECS). The JECS is an ongoing, nationwide prospective birth cohort study that aimed to recruit approximately 100000 pregnant women and their offspring; the recruitment period started in January 2011 and lasted until March 2014. The JECS has 15 regional centres (Hokkaido, Miyagi, Fukushima, Chiba, Kanagawa, Koshin, Toyama, Aichi, Kyoto, Osaka, Hyogo, Tottori, Kochi, Fukuoka and South Kyushu Okinawa). Each regional centre determined its own study area, consisting of one or more local administrative units (cities, towns or villages). The recruitment of pregnant women was community-based at the time of the first prenatal examination at cooperating healthcare providers (ie, obstetric facilities) or at local government offices issuing pregnancy journals. ${ }^{12}$ JECS aimed to cover half of all the births in the area, and the child coverage was approximately $45 \%$ in 2013. As a result, JECS covered around 3\% of Japanese newborns in 2013, and could be comparable with those obtained in the national survey. ${ }^{13}$ The detailed study profile of the JECS has been reported elsewhere. ${ }^{12} 13$

Biological samples and data are collected from the offspring of enrolled mothers from birth to the age of 13 years. Physicians, midwives/nurses and/or research coordinators collect relevant information (medical history including gravidity and related complications, parity, maternal anthropometry and infant physical examinations) from medical records. For this study, we used the JECS datasets jecs-ag-20160424 and jecs-ag-20160424-sp1, which were released in June 2016 and revised in October 2016. Written informed consent was obtained from all participants.

Maternal nutrition was assessed using a semiquantitative food frequency questionnaire $(\mathrm{FFQ})$ in the first trimester (FFQ_MT1). The questionnaire included a list of foods commonly consumed in Japan, as well as information on standard portion sizes. ${ }^{14}$ Participants were asked to report the daily, weekly or monthly frequencies of consumption and portion sizes of fermented foods. Using answers from the FFQ the daily intakes of nutrients were assessed for retinol $(\mu \mathrm{g})$, vitamin $\mathrm{D}(\mu \mathrm{g})$, $\alpha$-tocopherol $(\mathrm{mg})$, vitamin $\mathrm{K}(\mu \mathrm{g})$, vitamin $\mathrm{B}_{1}(\mathrm{mg})$, vitamin $\mathrm{B}_{2}(\mathrm{mg})$, niacin $(\mathrm{mg})$, vitamin $B_{6}(\mathrm{mg})$, vitamin $B_{12}(\mu \mathrm{g})$, folic acid $(\mu \mathrm{g})$, pantothenic acid $(\mathrm{mg})$, vitamin $\mathrm{C}(\mathrm{mg}), \omega-3$ unsaturated fatty acids $(\mathrm{g}), \omega-6$ unsaturated fatty acids $(\mathrm{g})$ and biotin $(\mu \mathrm{g})$. The maternal intake of supplements was assessed by using answers given in interviews regarding the use of drugs and supplements (InT1 (T1 means first trimester) and InT2 (T2 means in the second or third trimester)). Drug and supplement consumption were queried for three periods: before pregnancy confirmation, from the start of pregnancy up to week 12 (first trimester) and after week 12.

The study outcome was the diagnosed occurrence of CL, CP or CL with CP. These orofacial birth defects were evaluated within 1 month after birth by unit centres of the JECS across Japan by two paediatricians. Maternal age, body mass index at pregnancy, smoking status, alcohol intake, pregestational diabetes, retinol intake based on FFQ maternal education, folic acid intake during pregnancy, antirheumatic, antiepileptic or psychotropic drug intake during pregnancy, and children's sex were included as covariates potentially associated with orofacial clefts. Other variables, such as pregnancy history, marital status, job status of mothers, education of mothers and household income, were included for baseline analyses.

Prior to the main analysis, the association between maternal nutrient/supplement intake and CL, CP or CL with $\mathrm{CP}$ was explored; this preanalysis evaluation aimed to address a large number of considered nutrients. Intergroup comparisons for the occurrence of orofacial clefts were performed using the Kruskal-Wallis tests or the $\chi^{2}$ tests. Subsequently, multivitamin intake and outcomes were analysed in the main analysis. Baseline characteristics potentially associated with maternal supplement intake, the mother's age at pregnancy, marital status, job status, education and household income were assessed.

In the main analysis, multinomial logistic regression models were used to estimate the relative risk ratios (RRs) and $95 \%$ CIs; the RRs and CIs were used to characterise the associations between maternal multivitamin supplement intake and orofacial cleft diagnoses (CL, CP or CL with CP, respectively) at birth or 1 month after birth, and further analysed after adjusting for the abovementioned covariates. Multiple imputations were performed for missing values by chained equation (MICE) procedures. For sensitivity analyses, we evaluated a model in which missing values were imputed by Markov chain Monte Carlo (MCMC) procedures because some bias might have remained in the MICE simulation model. ${ }^{15}$ All $\mathrm{p}$ values were two-sided, and a p value of $<0.05$ was considered statistically significant. All statistical analyses were performed using STATA V.13.0 software (Stata Corp LP, College Station, Texas, USA).

\section{Patient and public involvement}

No cohort members were involved in setting the research question or outcome measures or in developing plans for the design or implementation of the study. No patients were involved in the interpretation or writing of results. There are no specific plans to disseminate the results of the research to cohort members, but dissemination to the general public will be undertaken by presentations and press releases.

\section{RESULTS}

The JECS included fetal records of 104102 children. Live birth information was available for 100148 (96.2\%) infants whose mothers did not withdraw their consent 
The JECS dataset included foetal records of 104102 children

$N=3954$

Mothers who withdrew consent $(\mathrm{N}=29)$

Mothers without a live birth or with missing birth

information data $(\mathrm{N}=3925)$

Mother and child pairs ( $N=100$ 148)

Mothers with missing nutrition data $(N=1361)$

Mother and child pairs included

in the analysis ( $\mathrm{N}=98787$ )

Figure 1 Flowchart of subject selection.

to participate in the JECS. After excluding children whose mothers reported no nutritional data, 98787 $(94.9 \%)$ mother-child pairs were included in the analysis (figure 1). The cohort included $50632(51.3 \%)$ boys and 48147 (48.7\%) girls (information on sex was missing for $0.05 \%$ of the children). Of the 98787 children, 69 $(0.07 \%)$ were diagnosed with cleft lip alone, $113(0.11 \%)$ were diagnosed with cleft lip and palate, and $52(0.05 \%)$ were diagnosed with cleft palate within 1 month after birth. A total of $234(0.24 \%)$ children were eventually diagnosed with CL, CP or CL with CP. This incidence was shown as 23.7 per 10000 births in our study.

Table 1 presents the results of exploratory analyses of the association between maternal nutrition status during the first trimester of pregnancy and CL, CP or CL with CP. No differences were observed in median nutrient intake estimated from FFQs among the CL with/without CP, CP and unaffected groups. However, multivitamin supplement intake during pregnancy differed between the groups without and with incidence of orofacial clefts. The characteristics of mothers and children and associations with multivitamin supplement intake are shown in table 2. The multivitamin supplement intake group had a slightly greater mean age at pregnancy (31.6 years vs 30.7 years) and higher frequency of first pregnancy $(37.7 \%$ vs $30.5 \%)$ than the no intake group. Furthermore, the multivitamin supplement intake group included larger proportions of women with full-time occupations $(34.7 \%$ vs $32.4 \%)$, higher levels of education (university or higher: $26.7 \%$ vs $20.9 \%$ ) and a higher level of income (>8 million yen (US\$72 500), $13.5 \%$ vs $9.70 \%$ ), than the no intake group.
Table 3 presents the results of the main analysis of the associations between multivitamin supplement intake and the incidence of CL, CP and CL with CP. Model 1 involved a crude analysis of these associations and found that multivitamin supplement intake before pregnancy was associated with orofacial clefts $(\mathrm{RR}=1.71 ; 95 \% \mathrm{CI} 1.06$ to 2.77), with a stronger association with intake up to the 12th week ( $R R=2.00 ; 95 \%$ CI 1.18 to 3.37$)$ than after the first trimester (RR=1.34; 95\% CI 0.59 to 3.01). Furthermore, the association between supplement intake up to week 12 and orofacial clefts remained significant after adjusting for the mother's age and other maternal variables during pregnancy (Model 2, RR=2.05, 95\% CI 1.21 to 3.46), as well as after adjusting for other variables, such as drug intake during pregnancy (Model 3, $\mathrm{RR}=2.11,95 \%$ CI 1.24 to 3.59).

Regarding the stratified outcome of CL with or without $\mathrm{CP}$, the relative risks were statistically significant for multivitamin intake before pregnancy confirmation (CL with CP, Model 2, RR=2.04, 95\% CI 1.06 to 3.92; Model 3, RR=2.05, 95\% CI:1.06 to 3.95) and during the first trimester (CL alone, Model 2, RR=2.80, 95\% CI 1.21 to 6.49; Model 3, $\mathrm{RR}=3.36,95 \%$ CI 1.43 to 7.90 ). Regarding the outcome of $\mathrm{CP}$ alone, we could not detect statistical significance for the association between multivitamin intake and the outcome in any period, although a trend towards this association was observed. Online supplementary table 1 shows the results of the model in which missing values were imputed by MCMC procedures. These sensitivity analyses did not reveal any change in the estimated relationship between maternal multivitamin intake and orofacial clefts in children. 
Table 1 Exploratory table of maternal nutrition status during the first trimester of pregnancy and the incidence of cleft palate with/without cleft lip ( $N=98787$ )

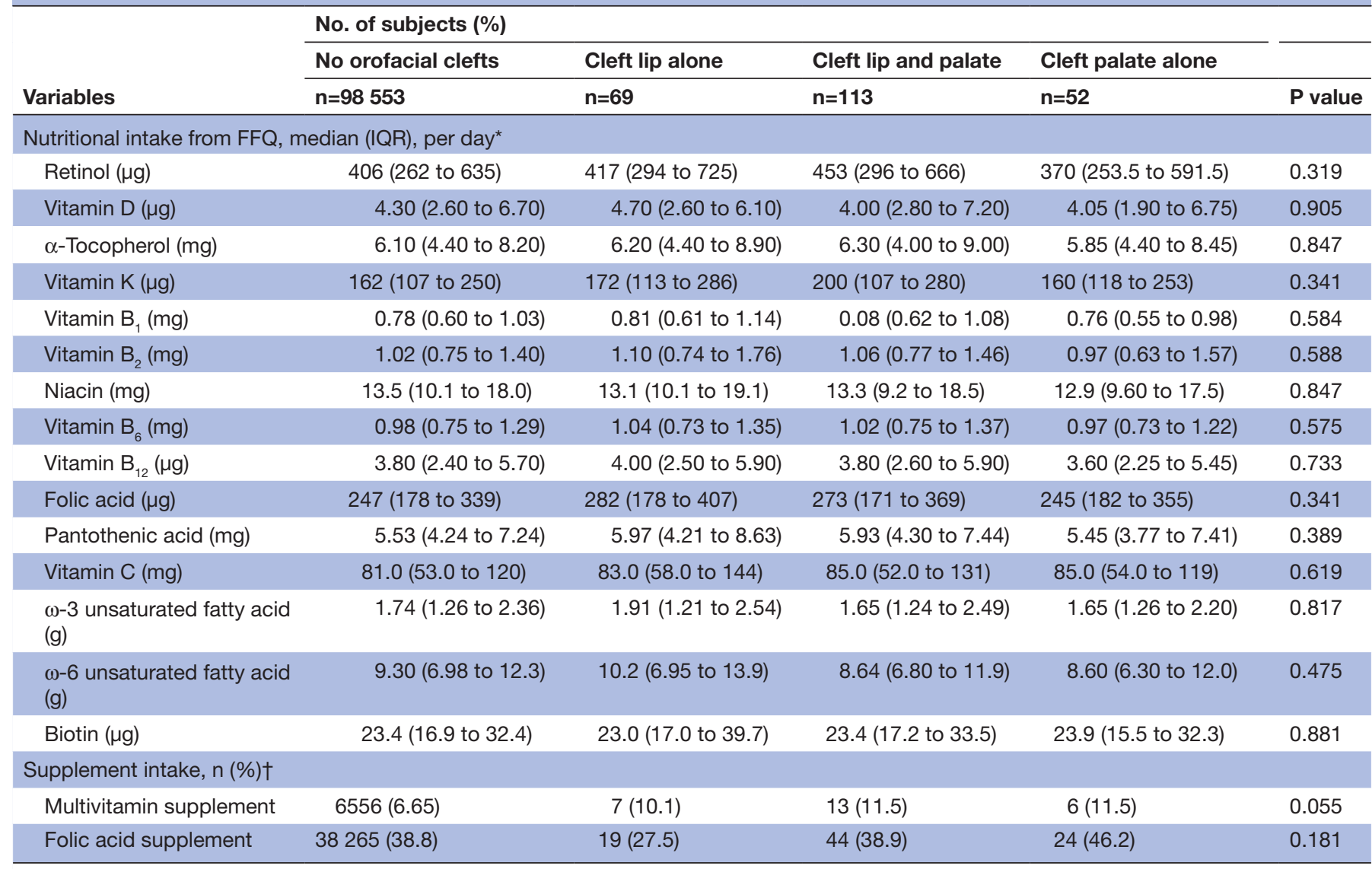

*Kruskal-Wallis tests were performed for comparison between groups.

$+\chi^{2}$ tests were performed for comparison between groups.

$\mathrm{FFQ}$, food frequency questionnaire.

\section{DISCUSSION}

The results of our analyses demonstrated an association between multivitamin supplement intake during pregnancy and the increased incidence of orofacial clefts. Specifically, compared with no intake of supplements, intake before pregnancy confirmation or intake during the first trimester of pregnancy was associated with a twofold increase in the relative risk of orofacial clefts in the offspring at birth. This association remained significant even after adjusting for maternal factors and the intake of specific drugs during pregnancy, although intake after 12 weeks failed to detect any significant association with orofacial clefts. Furthermore, micronutrient intake from food was not associated with the occurrence of CL with/without CP in the offspring.

A previous meta-analysis of maternal multivitamin use and adverse birth outcomes in a cohort of 13680 women with 1418 children with orofacial clefts, reported a summary relative risk (RR) of 0.88 (95\% CI 0.77 to 1.01$)$ for the association between periconceptional multivitamin use with CL with/without CP, and a summary RR of 1.12 (95\% CI 0.94 to 1.33 ) for CP alone. ${ }^{16}$ Among studies included in a meta-analysis, two studies found a significant decrease in the risk associated with intake of multivitamins with folic acid during pregnancy, ${ }^{17} 18$ whereas five studies failed to demonstrate any significant risk reduction. ${ }^{19-23}$ These discrepancies among the results of these studies may be attributable to the differences in the types of multivitamin supplements assessed as the studies focused mainly on folic acid-containing supplements.

In our study, our classification of any multivitamin use was only binary (yes or not), although different multivitamin supplements are available in Japan. Actually, multivitamin products sold in Japan are categorised as 'health food products', which are sold without regulation. Generally, multivitamin products sold in Japan contain vitamin A, vitamin $\mathrm{B}_{1}$, vitamin $\mathrm{B}_{2}$, vitamin $\mathrm{B}_{6}$, vitamin $\mathrm{B}_{12}$, niacin, pantothenic acid, biotin, folic acid, vitamin $\mathrm{C}$ and vitamin $\mathrm{E}$. As in other countries, women in the prepregnancy or initial pregnancy stage are recommended to take folic acid due to its preventive effect on neural tube defects in Japan. However, compared with purely folic acid supplement (100\% folic acid supplement), the dose of folic acid contained in multivitamins sold in Japan is not sufficient. If the women tend to take enough folic acid for prevention of neural tube defects from multivitamin, this may lead to overdose or excessive intake of other vitamins and minerals, which are reported as teratogens. For this purpose, the Food Safety 
Table 2 Characteristics of parents and children associated with multivitamin supplement intake (N=98 787$)$

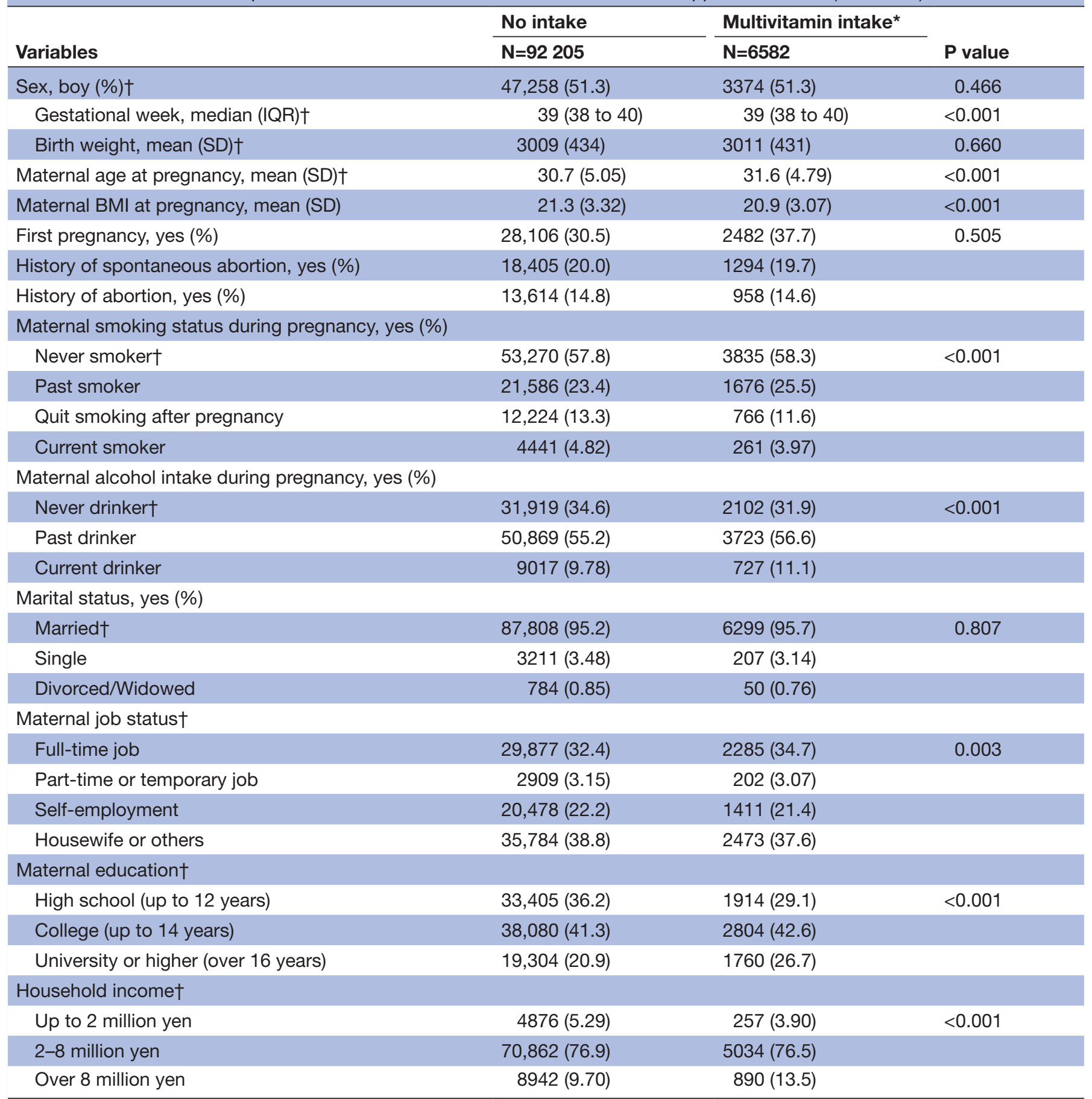

*Multivitamin intake means multivitamin intake during any period of 'before pregnancy confirmation', 'Up to 12 weeks' or 'After 12 weeks'. †Data were missing for sex for $8(0.01 \%)$, for birth weight for $68(0.07 \%)$, for mothers' age at pregnancy for $6134(6.21 \%)$, for mothers' BMI at pregnancy for $42(0.04 \%)$, for smoking status of mothers for $728(0.74 \%)$, for alcohol intake of mothers for $430(0.44 \%)$, for marital status for $428(0.43 \%)$, for job status of mothers for $3368(3.41 \%)$, for education of mothers for $1520(1.54 \%)$ and for household income for 7926 $(8.02 \%)$ subjects.

$\mathrm{BMI}$, body mass index.

Commission of Japanese government alerted that "Though multivitamins contain folic acid, which have preventive effects against neural tube defects, pregnant women should pay attention when they take multivitamin supplements because they contain vitamin A, which causes malformation at birth". ${ }^{24}$ However, this was only an official statement and does not prevail among the public because no official statement backs the use of pure folic acid for pregnant women instead of multivitamins in Japan. Due to the low level of knowledge of this effect among the public, the pregnant women in Japan may take in multivitamin supplements without knowledge of the risk of overdose. 
Table 3 Multinomial logistic regression analysis of maternal multivitamin intake and the incidence of cleft lip and cleft palate in children $(\mathrm{N}=98 \mathrm{787})$

\begin{tabular}{|c|c|c|c|c|}
\hline & No. of events (\%) & & & \\
\hline & Total orofacial clefts & Cleft lip alone & $\begin{array}{l}\text { Cleft lip with cleft } \\
\text { palate }\end{array}$ & $\begin{array}{l}\text { Cleft palate } \\
\text { alone }\end{array}$ \\
\hline & $\mathrm{N}=234$ & $\mathrm{n}=69$ & $n=113$ & $\mathrm{n}=52$ \\
\hline Variables & Risk ratio $(95 \% \mathrm{Cl})$ & Risk ratio $(95 \% \mathrm{Cl})$ & Risk ratio $(95 \% \mathrm{Cl})$ & $\begin{array}{l}\text { Risk ratio }(95 \% \\
\mathrm{Cl})\end{array}$ \\
\hline \multicolumn{5}{|l|}{ Model 1 (crude) } \\
\hline \multicolumn{5}{|c|}{$\begin{array}{l}\text { Multivitamin intake, reference Ref } \\
\text { (no) }\end{array}$} \\
\hline $\begin{array}{l}\text { Before pregnancy } \\
\text { confirmation }\end{array}$ & $1.71(1.06 \text { to } 2.77)^{\star}$ & 1.96 (0.85 to 4.52$)$ & 1.99 (1.04 to 3.82$)$ & $\begin{array}{l}0.82 \text { (0.20 to } \\
3.38)\end{array}$ \\
\hline $\begin{array}{l}\text { Up to } 12 \text { weeks (the first } \\
\text { trimester) }\end{array}$ & $2.00(1.18 \text { to } 3.37)^{\star}$ & $2.78(1.20 \text { to } 6.41)^{\star}$ & 1.35 (0.55 to 3.31$)$ & $\begin{array}{l}2.43(0.88 \text { to } \\
6.74)\end{array}$ \\
\hline After 12 weeks & $1.34(0.59$ to 3.01$)$ & $1.52(0.56$ to 6.19$)$ & $0.92(0.23$ to 3.71$)$ & $\begin{array}{l}2.03(0.49 \text { to } \\
8.36)\end{array}$ \\
\hline \multicolumn{5}{|l|}{ Model 2ł (adjusted) } \\
\hline \multicolumn{5}{|c|}{$\begin{array}{l}\text { Multivitamin intake, reference Ref } \\
\text { (no) }\end{array}$} \\
\hline $\begin{array}{l}\text { Before pregnancy } \\
\text { confirmation }\end{array}$ & $1.73(1.07 \text { to } 2.81)^{\star}$ & 1.93 (0.84 to 4.48$)$ & 2.04 (1.06 to 3.92$)^{\star}$ & $\begin{array}{l}0.84(0.20 \text { to } \\
3.45)\end{array}$ \\
\hline $\begin{array}{l}\text { Up to } 12 \text { weeks (the first } \\
\text { trimester) }\end{array}$ & 2.05 (1.21 to 3.46$)^{* *}$ & $2.80(1.21 \text { to } 6.49)^{*}$ & $1.40(0.57$ to 3.45$)$ & $\begin{array}{l}2.47(0.89 \text { to } \\
6.88)\end{array}$ \\
\hline After 12 weeks & 1.36 (0.60 to 3.07$)$ & 1.50 (0.37 to 6.13$)$ & 0.94 (0.23 to 3.83$)$ & $\begin{array}{l}2.08(0.50 \text { to } \\
8.60)\end{array}$ \\
\hline \multicolumn{5}{|l|}{ Model 3‡ (adjusted) } \\
\hline \multicolumn{5}{|c|}{$\begin{array}{l}\text { Multivitamin intake, reference Ref } \\
\text { (no) }\end{array}$} \\
\hline $\begin{array}{l}\text { Before pregnancy } \\
\text { confirmation }\end{array}$ & 1.77 (1.09 to 2.88$)^{*}$ & 2.21 (0.95 to 5.16$)$ & 2.05 (1.06 to 3.95$)^{\star}$ & $\begin{array}{l}0.77(0.19 \text { to } \\
3.19)\end{array}$ \\
\hline $\begin{array}{l}\text { Up to } 12 \text { weeks (the first } \\
\text { trimester) }\end{array}$ & 2.11 (1.24 to 3.59$)^{\text {t* }}$ & $3.36(1.43$ to 7.90$)$ & 1.39 (0.56 to 3.45$)$ & $\begin{array}{l}2.26(0.80 \text { to } \\
6.38)\end{array}$ \\
\hline After 12 weeks & 1.38 (0.61 to 3.13$)$ & 1.78 (0.43 to 7.34$)$ & 0.93 (0.23 to 3.79$)$ & $\begin{array}{l}1.89(0.45 \text { to } \\
7.86)\end{array}$ \\
\hline
\end{tabular}

${ }^{*} \mathrm{p}<0.05 ; \mathrm{p} \mathrm{p}<0.01$.

$\ddagger$ Adjusted for the mother's age and body mass index at pregnancy, smoking and alcohol consumption during pregnancy, pregestational diabetes, retinol intake based on FFQ (food frequency questionnaire) and maternal education.

$\S$ Adjusted for the mother's age and body mass index at pregnancy, smoking and alcohol consumption during pregnancy, pregestational diabetes, retinol intake based on FFQ, maternal education, folic acid intake during pregnancy, and antirheumatic or antiepileptic or psychotropic drug intake during pregnancy.

Our results suggest that the associations between multivitamin supplements and the incidence of orofacial clefts were largely observed during the first trimester, which is known to be the most drug-sensitive period of pregnancy. Fat-soluble vitamins, such as vitamin A, contained in multivitamins have been reported as teratogens, and these might play a potential role in the occurrence of orofacial clefts in offspring. ${ }^{25}$ Exposure to high doses of vitamin A might affect fetal palatogenesis by interfering with cell proliferation, as demonstrated in animal studies of neural tube closure and organ and limb development. ${ }^{26}{ }^{27}$ As another mechanism, there could be gene-environmental interaction between maternal multivitamin intake and genes in the aetiology of orofacial clefts. Actually, Wu et al have found gene and environmental interactions between maternal exposures to multivitamin supplementation and orofacial cleft among the Chinese population. ${ }^{28}$ However, their results showed preventive effect of maternal multivitamin supplementation and incidence of orofacial clefts. It is plausible to think that the adverse effect of multivitamin in our subjects could be caused by an overdose of teratogen nutrients taken during pregnancy. Although we could not confirm that the vitamin A in the supplements taken by mothers in our study caused orofacial clefts in the offspring, caution should be exercised regarding multivitamin overdose by pregnant women. 
The risks associated with excess micronutrient intake are less understood, compared with the risks and recommendations associated with insufficient micronutrition. Published evidence suggests that both nutritional excess and deficiency can lead to birth defects and intestinal malformations; however, the potential risks of excessive micronutrient intake have not been comprehensively determined..$^{29}$ The existing epidemiological evidence cannot be used to establish a clear threshold above which vitamin intake may be harmful during early pregnancy, ${ }^{30}$ and therefore, effective communication of risks should convey that folic acid has a protective effect on the embryo and fetus, whereas excess multivitamin intake may be detrimental.

This study involved a dataset from a nationwide, largescale cohort study, which allowed an adequate assessment of the outcome incidence. Although some studies conducted in Nordic countries have included large birth cohorts, ${ }^{31}$ to our knowledge, this is the first study to evaluate the association between multivitamin intake and orofacial clefts in the offspring in the largest Asian cohort recruited to date. As the prevalence of orofacial clefts is higher in Asian populations than in European populations, our study may be more relevant to Asian populations.

Our study had several limitations. First, the data on maternal intake of multivitamin supplements and other drugs were based on interviews, which might have led to misclassification of exposure due to self-report bias. Furthermore, we did not investigate the types of multivitamin supplements used, and therefore, the components and doses were unknown. Accordingly, the specific effects of vitamin A and other micronutrients on the incidence of orofacial clefts in the offspring could not be determined. Second, we only considered the occurrence of CL, CP or CL with CP within 1 month after birth. Thus, it is possible that we may have excluded CP cases which could not be diagnosed within this period. Additionally, in a stratified analysis, we did not observe a clear association between multivitamin intake during pregnancy and the incidence of $\mathrm{CP}$ alone. This finding may be explained by a previous observation that CL and CP have different aetiologies. ${ }^{32}$ On the other hand, 52 occurrences may not have been sufficient for a thorough statistical analysis. The outcome of our study was rare; therefore, larger cohorts or a well-designed case-control study where exposures will be captured accurately, including information on the content of multivitamin supplements, would be necessary in future. Third, we could not fully adjust for all potential confounding factors, including siblings and hereditary factors, which may have had an impact on the occurrence of CL, CP or CL with CP. Fourth, our outcome measure did not consider syndromic and non-syndromic cases. Although JECS data contain the information about congenital malformation in children, it is difficult to distinguish between syndromic or non-syndromic CL/ CP because we could not check all congenital malformations related to CL/CP. Finally, our study only considered liveborn children, and this can occur in a potential survival bias for pregnancies. This means multivitamins may increase the survival of a fetus with an orofacial cleft to birth rather than a risk due to the multivitamin. These limitations suggest that further studies should consider the components of multivitamin supplements and their effects on the incidence of orofacial clefts.

\section{CONCLUSION}

Our study demonstrated that the maternal intake of multivitamin supplements during early pregnancy increased the risk of orofacial clefts in the offspring, whereas micronutrient intake from food was not associated with such occurrence. Notably, this association was observed during the first trimester of pregnancy but was not significant thereafter. Accordingly, pregnant women and those intending to become pregnant should be informed about the benefits of taking pure folic acid supplements and advised about the potential risks of excess multivitamin supplement intake. Even though vitamin deficiency negatively affects fetal development, the excessive intake of multivitamin supplements could result in congenital malformations.

Acknowledgements We thank all participants from the JECS group and all individuals involved in data collection. Members of JECS as of 2018: Japan Industrial Safety \& Health Association, Tokyo: Toshihiro Kawamoto (principal investigator); Medical Support Centre for JECS, National Centre for Child Health and Development, Tokyo: Yukihiro Ohya; Hokkaido Regional Centre for JECS, Hokkaido University, Sapporo: Reiko Kishi; Miyagi Regional Centre for JECS, Tohoku University, Sendai: Nobuo Yaegashi; Fukushima Regional Centre for JECS, Fukushima Medical University, Fukushima: Koichi Hashimoto; Chiba Regional Centre for JECS, Chiba University, Chiba: Chisato Mori; Kanagawa Regional Centre for JECS, Yokohama City University, Yokohama: Shuichi Ito; Koshin Regional Centre for JECS, University of Yamanashi, Chuo: Zentaro Yamagata; Toyama Regional Centre for JECS, University of Toyama, Toyama: Hidekuni Inadera; Aichi Regional Centre for JECS, Nagoya City University, Nagoya: Michihiro Kamijima; Kyoto Regional Centre for JECS, Kyoto University, Kyoto: Takeo Nakayama; Osaka Regional Centre for JECS, Osaka University, Suita: Hiroyasu Iso; Hyogo Regional Centre for JECS, Hyogo College of Medicine, Nishinomiya: Masayuki Shima; Tottori Regional Centre for JECS, Tottori University, Yonago: Yasuaki Hirooka; Kochi Regional Centre for JECS, Kochi University, Nankoku: Narufumi Suganuma; Fukuoka Regional Centre for JECS, University of Occupational and Environmental Health, Kitakyushu: Koichi Kusuhara; South Kyushu/Okinawa Regional Centre for JECS, Kumamoto University, Kumamoto: Takahiko Katoh.

Collaborators Japan Environment and Children's Study Group.

Contributors All authors fulfil the criteria for authorship; JECS, SY, MT, CK, KK and SI initiated the study; CK and JECS collected the data; SY and MT analysed the data; SY, MT, CK, KK and SI interpreted the data. All authors commented on the draft and have seen and approved the final version. The corresponding author attests that all listed authors meet authorship criteria and that no others meeting the criteria have been omitted.

Funding The JECS was funded by the Ministry of the Environment of Japan. The funder had no role in formulating the study design, collection, analysis and interpretation of data, writing of the report or in the decision to submit the paper for publication. Furthermore, the findings and conclusions of this article are solely the responsibility of the authors and do not represent the official views of the government of Japan. The corresponding author had full access to the data and had final responsibility for the decision to submit for publication.

Competing interests Japan Environment and Children's Study Group.

Patient and public involvement Patients and/or the public were not involved in the design, or conduct, or reporting or dissemination plans of this research. 
Patient consent for publication Not required.

Ethics approval The JECS protocol was approved by the Institutional Review Board on Epidemiological Studies of the Ministry of the Environment, and the ethics committees of all participating institutions. This study protocol was approved by the ethics committee of the Kyoto University Graduate School and the Faculty of Medicine (R1131).

Provenance and peer review Not commissioned; externally peer reviewed.

Data availability statement № data are available. № additional data were available.

Open access This is an open access article distributed in accordance with the Creative Commons Attribution Non Commercial (CC BY-NC 4.0) license, which permits others to distribute, remix, adapt, build upon this work non-commercially, and license their derivative works on different terms, provided the original work is properly cited, appropriate credit is given, any changes made indicated, and the use is non-commercial. See: http://creativecommons.org/licenses/by-nc/4.0/.

\section{ORCID iD}

Koji Kawakami http://orcid.org/0000-0002-7477-4071

\section{REFERENCES}

1 Mossey PA, Little J, Munger RG, et al. Cleft lip and palate. Lancet 2009;374:1773-85

2 Mai CT, Cassell CH, Meyer RE, et al. Birth defects data from population-based birth defects surveillance programs in the United States, 2007 to 2011: highlighting orofacial clefts. Birth Defects Research Part A: Clinical and Molecular Teratology 2014;100:895-904.

3 IPDTOC Working Group. Prevalence at birth of cleft lip with or without cleft palate: data from the International perinatal database of typical oral clefts (IPDTOC). Cleft Palate Craniofac J 2011;48:66-81.

4 Holmes LB, Baldwin EJ, Smith CR, et al. Increased frequency of isolated cleft palate in infants exposed to lamotrigine during pregnancy. Neurology 2008;70:2152-8.

5 Tomson T, Battino D. Teratogenic effects of antiepileptic drugs. Lancet Neurol 2012;11:803-13.

6 Honein MA, Rasmussen SA, Reefhuis J, et al. Maternal smoking and environmental tobacco smoke exposure and the risk of orofacial clefts. Epidemiology 2007;18:226-33.

7 DeRoo LA, Wilcox AJ, Lie RT, et al. Maternal alcohol binge-drinking in the first trimester and the risk of orofacial clefts in offspring: a large population-based pooling study. Eur J Epidemiol 2016;31:1021-34.

8 Hernández-Díaz S, Werler MM, Walker AM, et al. Folic acid antagonists during pregnancy and the risk of birth defects. $N$ Engl J Med 2000;343:1608-14.

9 Johnson CY, Little J. Folate intake, markers of folate status and oral clefts: is the evidence converging? Int J Epidemiol 2008;37:1041-58.

10 Krapels IPC, van Rooij IALM, Ocké MC, et al. Maternal nutritional status and the risk for orofacial cleft offspring in humans. J Nutr 2004;134:3106-13.

11 Vujkovic M, Ocke MC, van der Spek PJ, et al. Maternal Western dietary patterns and the risk of developing a cleft lip with or without a cleft palate. Obstet Gynecol 2007;110:378-84.

12 Kawamoto T, Nitta $\mathrm{H}$, Murata K, et al. Rationale and study design of the Japan environment and children's study (JECS). BMC Public Health 2014;14:25.
13 Michikawa T, Nitta H, Nakayama SF, et al. Baseline profile of participants in the Japan environment and children's study (JECS). $J$ Epidemiol 2018;28:99-104.

14 Yokoyama Y, Takachi R, Ishihara J, et al. Validity of short and long self-administered food frequency questionnaires in ranking dietary intake in middle-aged and elderly Japanese in the Japan public health Center-Based prospective study for the next generation (JPHC-NEXT) protocol area. J Epidemiol 2016;26:420-32.

15 Kim S, Sugar CA, Belin TR. Evaluating model-based imputation methods for missing covariates in regression models with interactions. Stat Med 2015;34:1876-88.

16 Wolf HT, Hegaard HK, Huusom LD, et al. Multivitamin use and adverse birth outcomes in high-income countries: a systematic review and meta-analysis. Am J Obstet Gynecol 2017;217:404. e1-404.e30.

17 Shaw GM, Lammer EJ, Wasserman CR, et al. Risks of orofacial clefts in children born to women using multivitamins containing folic acid periconceptionally. Lancet 1995;346:393-6.

18 Itikala PR, Watkins ML, Mulinare J, et al. Maternal multivitamin use and orofacial clefts in offspring. Teratology 2001;63:79-86.

19 Hayes C, Werler MM, Willett WC, et al. Case-Control study of periconceptional folic acid supplementation and oral clefts. Am J Epidemiol 1996;143:1229-34.

20 Werler MM, Hayes C, Louik C, et al. Multivitamin supplementation and risk of birth defects. Am J Epidemiol 1999;150:675-82.

21 Czeizel AE, Dobó M, Vargha P. Hungarian cohort-controlled trial of periconceptional multivitamin supplementation shows a reduction in certain congenital abnormalities. Birth Defects Res A Clin Mol Teratol 2004;70:853-61.

22 Shaw GM, Carmichael SL, Laurent C, et al. Maternal nutrient intakes and risk of orofacial clefts. Epidemiology 2006;17:285-91.

23 Wilcox AJ, Lie RT, Solvoll K, et al. Folic acid supplements and risk of facial clefts: national population based case-control study. BMJ 2007;334:464.

24 Food Safety Commission in Japan. Available: http://www.fsc.go.jp/ osirase/kenkosyokuhin.data/kenkosyokuhin_datakenkosyokuhin_ houkoku.pdf

25 Martínez-Frías ML, Salvador J. Epidemiological aspects of prenatal exposure to high doses of vitamin A in Spain. Eur J Epidemiol 1990;6:118-23.

26 Ackermans MMG, Zhou H, Carels CEL, et al. Vitamin A and clefting: putative biological mechanisms. Nutr Rev 2011;69:613-24.

27 Abbott BD, Harris MW, Birnbaum LS. Etiology of retinoic acidinduced cleft palate varies with the embryonic stage. Teratology 1989;40:533-53.

28 Wu T, Liang KY, Hetmanski JB, et al. Evidence of gene-environment interaction for the IRF6 gene and maternal multivitamin supplementation in controlling the risk of cleft lip with/without cleft palate. Hum Genet 2010;128:401-10.

29 Bruins MJ, Kupka R, Zimmermann MB, et al. Maximizing the benefits and minimizing the risks of intervention programs to address micronutrient malnutrition: symposium report. Matern Child Nutr 2016;12:940-8.

30 Dolk HM, Nau H, Hummler H, et al. Dietary vitamin A and teratogenic risk: European teratology Society discussion paper. Eur J Obstet Gynecol Reprod Biol 1999;83:31-6.

31 Gildestad T, Bjørge T, Vollset SE, et al. Folic acid supplements and risk for oral clefts in the newborn: a population-based study. $\mathrm{Br} J$ Nutr 2015;114:1456-63.

32 Meng L, Bian Z, Torensma R, et al. Biological mechanisms in palatogenesis and cleft palate. J Dent Res 2009;88:22-33. 